\title{
The Effect of Mutation in Brazzein Deduced from Mutational Sites and Sequence Carbon Content
}

\author{
Ezekiel Amri \\ Department of Science and Laboratory Technology, Dares Salaam Institute of Technology, Dares Salaam, Tanzania
}

Email address:

ezekielamri@yahoo.com

To cite this article:

Ezekiel Amri. The Effect of Mutation in Brazzein Deduced from Mutational Sites and Sequence Carbon Content. International Journal of Genetics and Genomics. Vol. 3, No. 6, 2015, pp. 59-65. doi: 10.11648/j.ijgg.20150306.11

\begin{abstract}
Brazzein is a small sweet-tasting protein isolated from the fruit of the African plant, Pentadiplandra brazzeana Baillon with potential of replacement of carbohydrate sweeteners. Carbon content analysis was used to examine the effect of mutation in brazzein's two regions at residues 29-33 and 39-43 with residue 36 reported to be important in sweet tasting of the protein. Analysis for local carbon density at the mutational sites for brazzein mutants with increased sweetness taste at residues 29 and 41 revealed normal carbon distribution curves with increased carbon frequency peak compared to the wild-type, consequently stabilized the local structure. Brazzein mutants with reduced sweetness taste at residue position 30, 33, 36 and 43 were mostly characterized by abnormal broadened distribution curve for carbon content with decreased frequency peak which destabilized the local structure and possibly leading to loss of protein functionality. Further analysis of carbon distribution profile along protein sequences of brazzein revealed a variation in carbon distribution between mutants with increased sweetness taste and those with decreased sweetness taste. Mutants with increased sweetness taste had carbon distribution profile balancing well conforming to the globular proteins which prefers to have $31.45 \%$ of carbon all along the sequence for stability. This study has provided further information and additional insights into protein atomic composition in brazzein and its role in understanding the effect of mutation.
\end{abstract}

Keywords: Brazzein, Carbon Content, Mutation, Sweet-Tasting Protein

\section{Introduction}

Brazzein is the smallest sweet-tasting protein consisting of 54 amino-acid residues and four disulfide bonds with a molecular mass of approximately $6.5 \mathrm{kDa}$, with good stability at high temperatures and over a wide $\mathrm{pH}$ range. Brazzein was isolated from the fruit of wild African plant Pentadiplandra brazzeana Baillon [1, 2]. Brazzein has inherent sweetness 500 to 2000 times that of sucrose on a molar basis with reported different uses such as tastemodifying protein and as an alternative to low calorie sweeteners [3].

There is a high demand for low calorie, protein-based sweeteners with promising taste properties. Naturally occurring sweet and taste modifying proteins are being seen as potential replacements for the carbohydrate sweeteners. Sweetness from carbohydrates such as sugars have several problems associated with their use ranging from tooth decay to diabetes mellitus. As a sweet-tasting protein, brazzein has the potential for replacement sugars, by acting as natural and high-quality sweeteners. Brazzein can be blended with other sweeteners such as aspartame and stevia and complements their taste $[4,5]$. Brazzein has been used in fruits, vegetables and grains for sweetened flour to amplify their sweetness [5, 6]. Other valuable uses of brazzein include antimicrobial activity against bacteria and the fungus [7].

The function of a protein mainly depends on its amino acids sequence and stability. Mutation of amino acid sequence can occur with or without effects on protein stability and function. Previous studies on mutations in brazzein have reported some effects on its sweet- tasting properties whereby some mutations have resulted in largest increase in sweetness, while other mutations have basically caused brazzein to be tasteless [1,8-11]. The studies have examined the role of the surface charge of the molecule as being an important for brazzein's sweetness taste. The role of carbon content and its distribution along in protein sequences has rarely been investigated in relation to mutation in proteins although carbon is the main architectural component of proteins. Amino acids can vary significantly in their contents of specific individual atoms such as carbon which 
has a potential of revealing hydrophobic and hydrophilic regions in the protein [12-14]. The purpose of the current study was to investigate how the carbon content distribution is useful in examining brazzein protein stability and its function upon mutations.

\section{Materials and Methods}

The protein structure and sequence of brazzein was downloaded from the Protein Data Bank (PDB) available at [www.rcsb.org] with the PDB identifier 4HE7. Brazzein mutants used in this study are based on mutagenesis studies in brazzein's two important regions previously reported as determinants of sweetness taste of the protein at residues 29 33 and 39-43, plus residue 36 [1, 8, 9]. The residues specifically examined were in position number $29,30,31,33$, 36,41 and 43. In order to elucidate the variation between mutants with increased brazzein sweetness and the mutants with decreased brazzein sweetness, analysis for local carbon density at the mutational sites was done along brazzein protein sequences for the mutants using a protein sequence CARD program for carbon $[12,13]$.The carbon content distribution profile outputs of Carbana and sequence CARD were then plotted for better visualization of results. For the analysis of the carbon distribution profile for brazzein sequences both the wild-type and mutants, the Carbana software available online (www.rajasekaran.net.in/tools/carbana.html) was used. The protein sequence in FASTA format is converted into atomic sequence through Carbana program then statistically analyzed for carbon content. The carbon distribution profile obtained was used to locate the hydrophobic and hydrophilic regions along the protein sequence for the wild-type and mutants. Amino acid composition for brazzein was also computed online through http://web.expasy.org/cgibin/protparam/protparam and source for brazzein structure from http://www.ebi.ac.uk/pdbsum/.

\subsection{Local Carbon Density at the Mutational Sites of Brazzein Mutants with Increased Sweetness}

Figure 1 and 2 shows the local carbon density of brazzein mutants at the mutational sites with increased sweetness taste. The normal local carbon distribution curve for normal and stable protein should be centered at 0.314 . Higher value to 0.314 is an indication that the stretch is in hydrophobic while lower of this value is an indication that the stretch is in hydrophilic region. For residue 29 the carbon distribution at mutational sites D29A, D29N and D29K are given in figure 1. For mutation site D29A the mutant has stretch carbon content in hydrophilic region at 0.257 with considerably increased frequency peak compared to the wild-type which has a stretch of carbon content in hydrophilic region at 0.286 . The mutant D29A has higher carbon frequency peak than the wide type in the same hydrophilic region which is stabilizing the local structure.

In the same residue 29 , the mutant $\mathrm{D} 29 \mathrm{~N}$ has the same carbon content distribution curve as that of wild-type at 0.286 but mutant has also higher carbon frequency peak than the wild-type which stabilizes the local structure. This kind of mutation only resulted in charge silencing as it causes change in charge only, not hydrophobic characters and leading towards an equal in number of carbon for the residues which doesn't affect the stability of protein because of the same carbon content in the side chains.

For the mutant D29K, the carbon content distribution curve is at 0.343 in hydrophobic region with significantly increased height of frequency peak compared to the wildtype which has a carbon distribution curve at 0.286 in hydrophilic region. The stretch of mutant in hydrophobic region with increased height of carbon frequency peak helps to stabilize the local structure. It should be noted that the stretch at this mutation is from small residue Asp to larger residue Lys therefore adds carbon and stabilizes the local structure.

\section{Results}
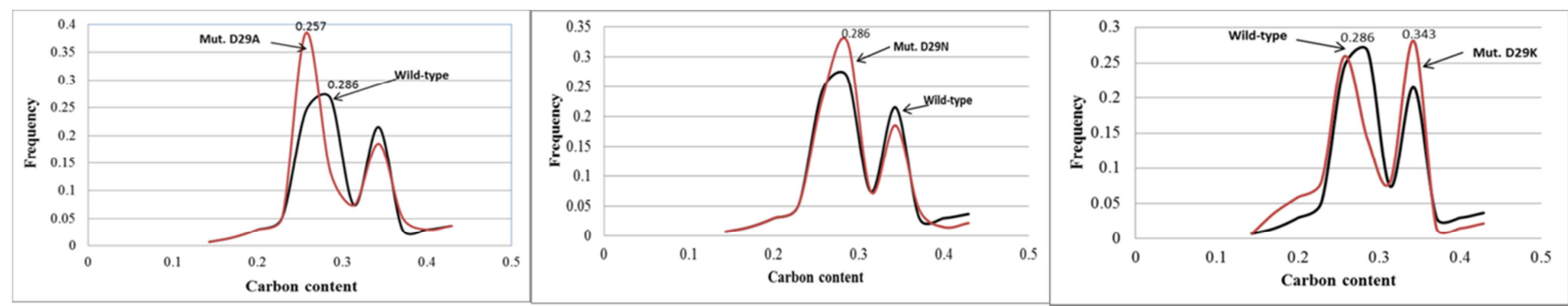

Figure 1. Carbon density for brazzein at residue 29 for mutational sites D29A, D29N and D29K.

Carbon distribution at mutational sites H31A, E41K and E41Q is given in Figure 2. For mutation site H31A the carbon content distribution curve of the mutant was towards hydrophobic region 0.329 marked also with increased height of carbon frequency peak compared to the wild-type which had value at 0.314 but with decreased frequency peak. At the mutation site $\mathrm{E} 41 \mathrm{~K}$, the mutation adds carbon resulting with stretch of carbon distribution curve at 0.286 which is the same as that of wild-type in hydrophilic region. Although the mutant and the wild-type have the same value for carbon distribution curve but the mutant has slightly high frequency peak compared to the wild-type which is very important in stabilizing the local structure.

At the mutation site E41Q the mutant does not alter the distribution curve, the mutant and the wild-type have the same shape and size for carbon content distribution curve at 
0.314 which is a value for a stable protein. This mutation has also resulted in charge silencing as it causes change in charge only, not hydrophobic characters. With its value at 0.314 , the mutant doesn't affect the stability of protein after the mutation and maintains the same carbon content in the side chains.
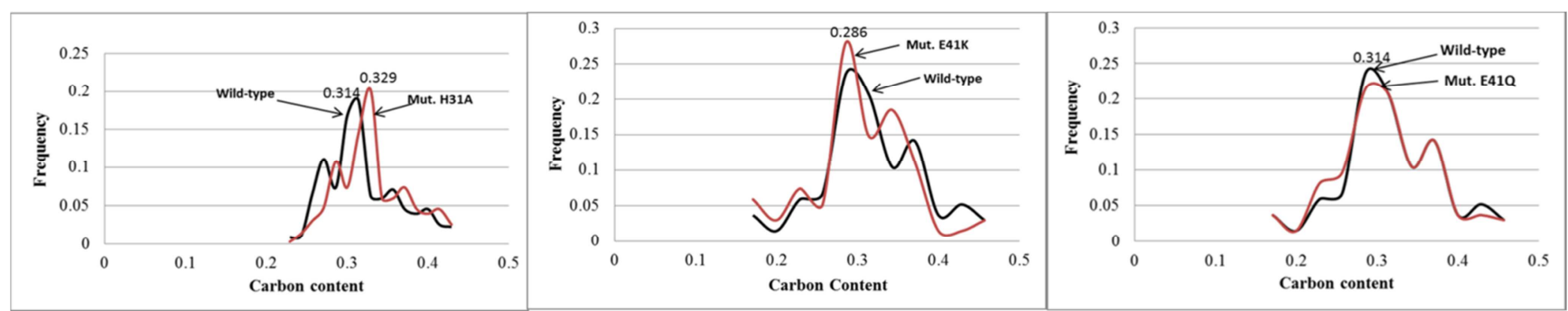

Figure 2. Carbon density for brazzein at residues 31 and 41 for mutational sites $H 31 A$, E41K and E41Q.

\subsection{Local Carbon Density at Mutational Sites of Brazzein Mutants with Reduced Sweetness}

Figure 3, 4 and 5 show different mutational sites at residues 30, 33, 36 and 43 of brazzein mutants with reduced sweetness taste. Substantial change in carbon content distribution is observed for mutants in this category compared to the brazzein mutants with increased sweetness taste. Mutational sites at residue 30 and 33 are shown in Figure 3. At residue 30 for mutant K30D the distribution curve of carbon content of mutant is at 0.329 in hydrophobic region with significantly decreased height of carbon frequency peak that destabilizes the local structure. The wildtype has value at 0.314 of stable protein, therefore the mutant's shifting from this value also destabilize the local structure of the protein. At residue 33 for mutation site R33A, the mutant and wild-type have the same value for the carbon content distribution curve at 0.286 in the hydrophilic region. This mutation is marked with decreased number of carbon fraction of the side chain resulting in significantly decreased height of carbon frequency peak which destabilizes protein local structure.

At residue 36 there are three mutants namely E36A, E36Q and E36K indicated in Figure 4. The mutant E36A has a widened carbon content distribution curve with significantly decreased frequency peak in hydrophobic region at 0.371 . The mutant is shifting from hydrophilic region at 0.286 of wild-type which has high carbon frequency peak. Although the mutant distribution curve moves to hydrophobic region, the widening of the distribution curve and shortening height of frequency peak destabilize the protein local structure.

At mutation site E36Q both mutant and wild-type have an equal in number of carbon for the residues and the carbon content distribution curve of the mutant and the wild-type is the same in hydrophilic region at 0.286 . However, the mutant has a significantly decreased carbon frequency peak compared to the wild-type which destabilizes the local structure of the protein. At residue position 36 for the mutant E36K, the wild-type has a high distribution curve in hydrophilic region at 0.286 and the mutant had value at 0.314 . Despite the fact that the mutant attained distribution values considered to be stable at 0.314 , it has a lower decreased carbon frequency peak and broadened distribution curve than the wild-type thus destabilizes the local structure.

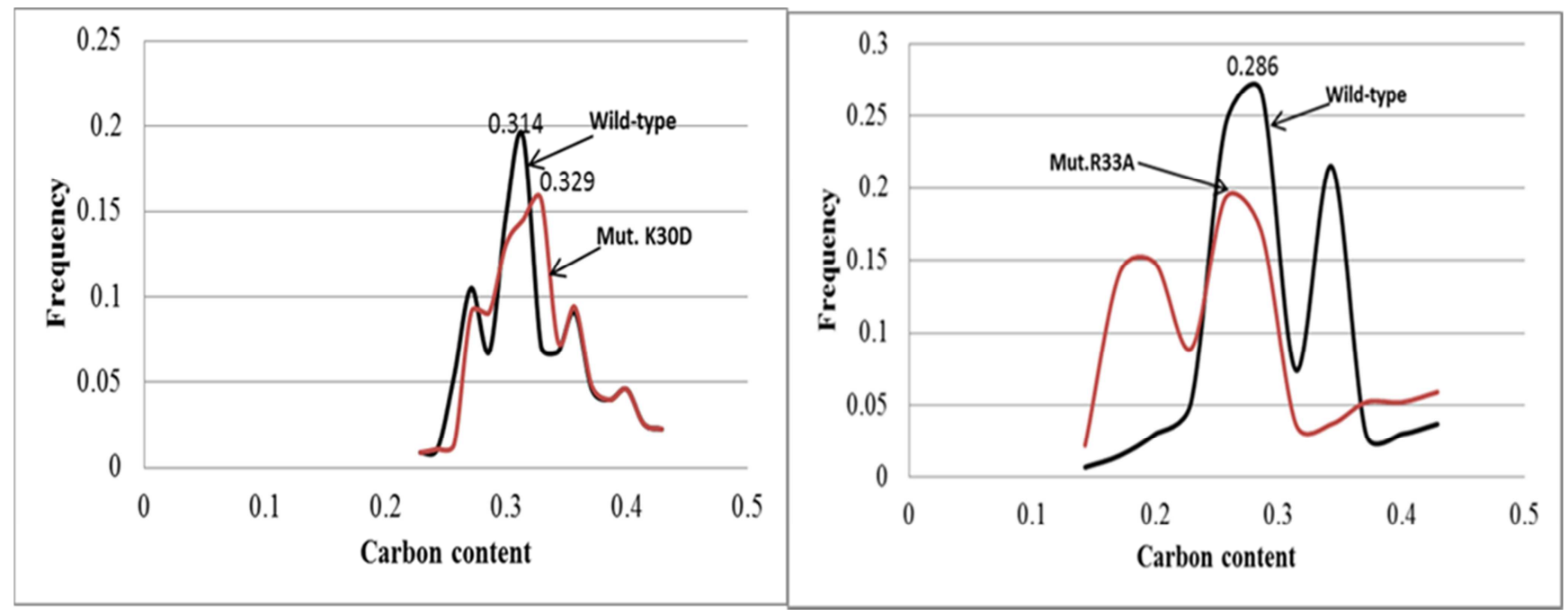

Figure 3. Carbon density for brazzein at residues 30 and 33 for mutational sites K3OD and R33A. 


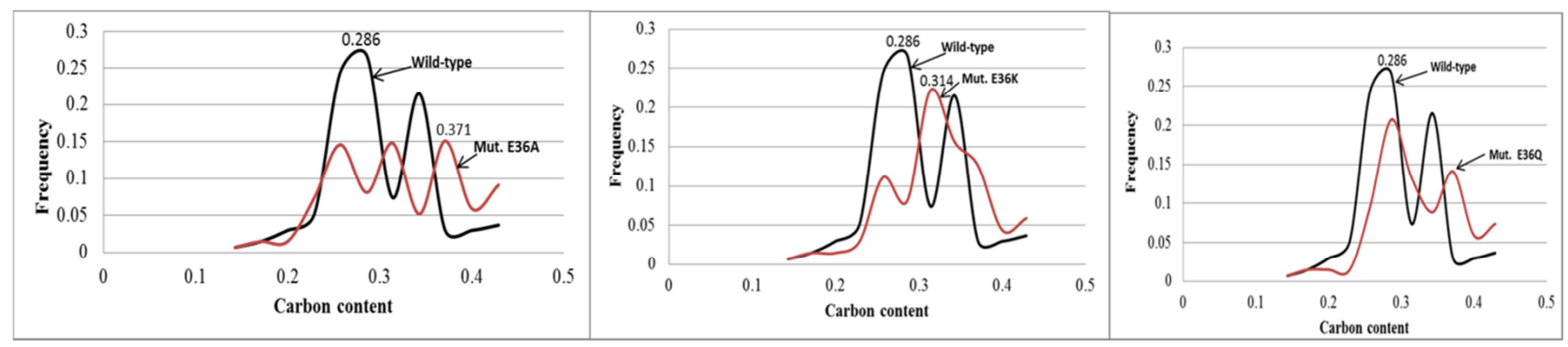

Figure 4. Carbon density for brazzein at residue 36 for mutational sites E36A, E36Q and E36K.
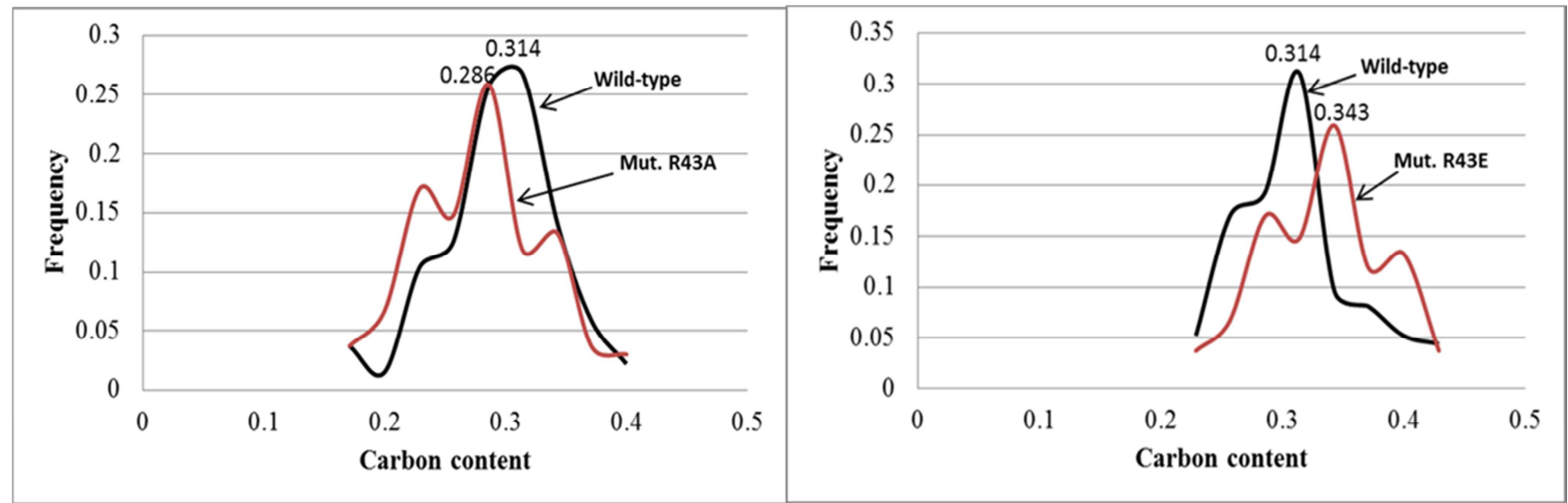

Figure 5. Carbon density for brazzein at residue 43 for mutational sites $R 43 A$ and $R 43 E$.

For mutants at residue 43 namely R43A and R43E are shown in Figure 5. At mutation site R43A, the mutant is shifting a carbon distribution curve towards hydrophilic region at 0.286 from 0.314 of stable normal distribution curve of the wild-type. The mutant has a decreased carbon frequency peak compared to the wild-type and such shifting of away from the normal distribution curve of a stable protein destabilizes the protein local structure. At mutation site R43E the mutant has a carbon content distribution curve in hydrophobic region at 0.343 with decreased height of frequency peak compared to the wild-type which has a value for stable structure at 0.314 . The shifting of mutant from stable values at 0.314 of the wild-type towards hydrophobic region with significantly decreased frequency peak destabilizes the local structure.

\subsection{Carbon Distribution Profile Variation for Protein Sequences of Brazzein Mutants}

Variation in carbon distribution profile for protein sequences of brazzein mutants with increased brazzein sweetness and mutants with reduced brazzein sweetness was given in Figure 6. Normally the carbon distribution profile for globular proteins prefers to have $31.45 \%$ of carbon all along the sequence for its stability. The middle horizontal line of each separate graph represents the optimum carbon content $(31.45 \%)$ for the stable protein. The values above $31.45 \%$ are considered to be higher carbon content or hydrophobic regions. As shown in Figure 6, the wild-type has the percentage of carbon is well maintained at $31.45 \%$ all along the brazzein sequence indicating that the hydrophobicity balance quite well for the entire sequence of the wild-type as a stable protein.

The carbon distribution profiles for the sequences of brazzein mutants with increased sweetness taste (D29A and E41K) are given in Figure 6 (a). The protein sequences for these mutants have normal distribution of carbon at $31.45 \%$ which balance well all along their sequences just like that of the wild- type despite the mutations. The balancing well for the overall hydrophobicity of all the proteins wild-type and mutants in their entire sequence is an indication that despite the mutation in at these key regions, the protein is still stable and maintaining its functions. The carbon distribution profile for the sequences brazzein mutants with reduced brazzein sweetness (K30D, R43E) are given in Figures 6 (b). The carbon distribution profiles along the sequences of brazzein mutants with reduced sweetness taste do not balance well as compared to mutants with increased sweetness taste. Most of the portions $(>60 \%)$ are below $31.45 \%$, the value considered being low carbon content or hydrophilic regions thus indicating that they are unstable proteins.

\subsection{Amino Acid Composition and Structure of Brazzein}

Figure 7 shows the amino acid percentage composition in brazzein, predominantly occupied by Cysteine (Cys), Lysine (Lys) and Tyrosine (Tyr) in a total of 54 amino acid residues. The following residues are absent in brazzein as part of its composition Methionine (Met), Threonine (Thr) and Tryptophan (Trp). The crystal structure of brazzein 4HE7 with 54 amino-acid residues as reported by [2] is given in 
Figure 8 . The structure shows that the protein has one alphahelix and three strands of anti-parallel beta sheet linked by
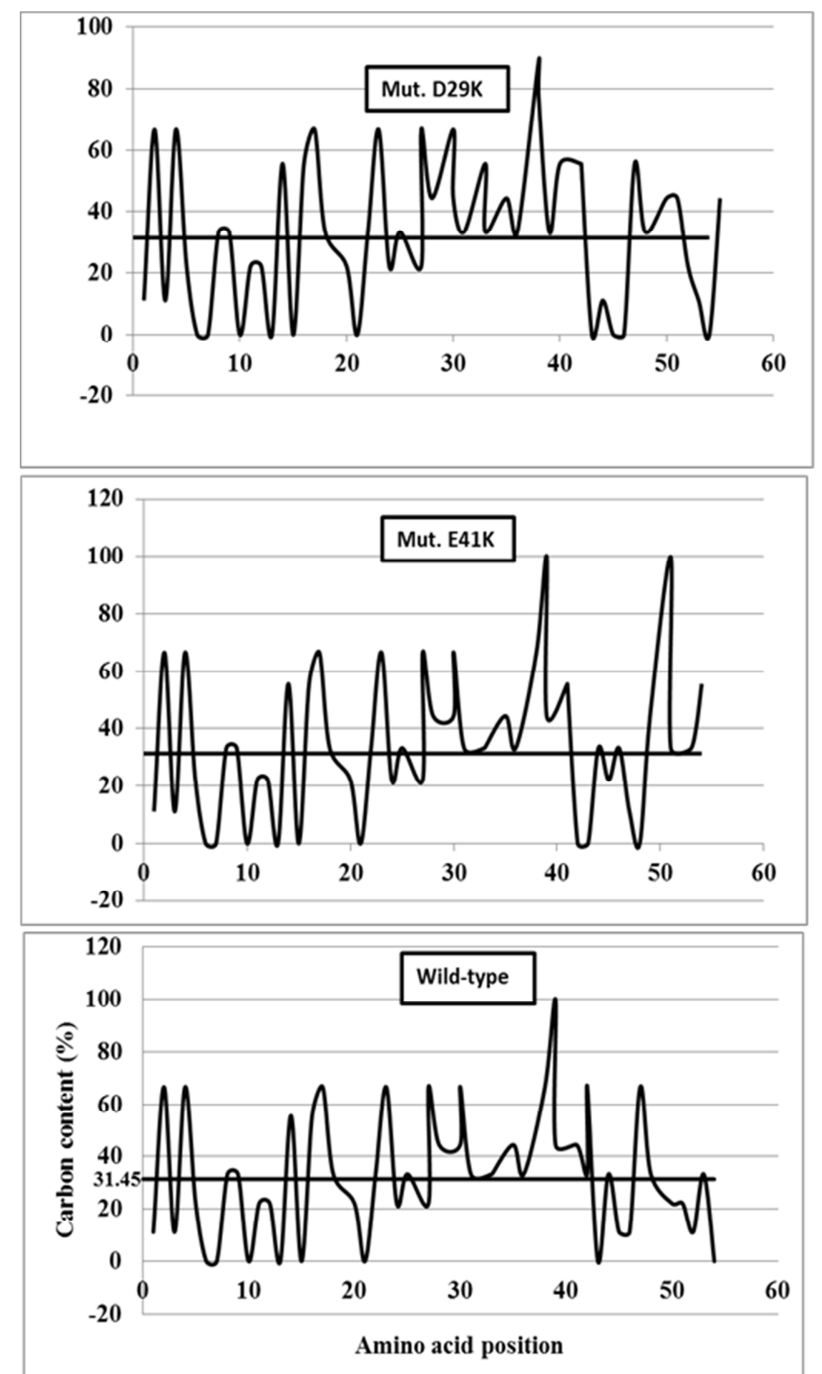

A: Profile for sequences of Brazzein mutants with increased sweetness backbone hydrogen bonds and four equally spaced disulfide bonds that sustain the structure in a stable conformation.
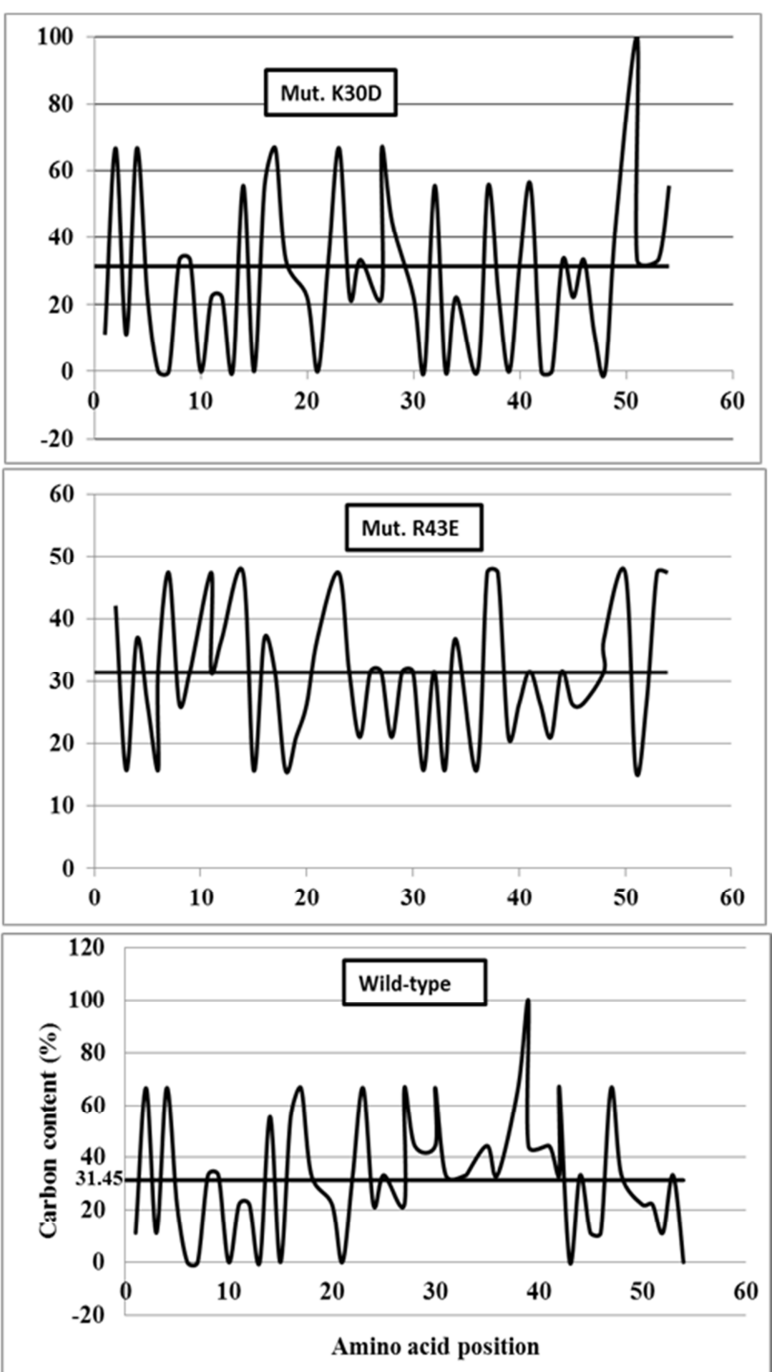

B: Profile for sequences of Brazzein mutants with reduced sweetness

Figure 6. Carbon distribution profile variation between (a) sequences of brazzein mutants with increased sweetness taste and (b) sequences of brazzein mutants with reduced sweetness taste.

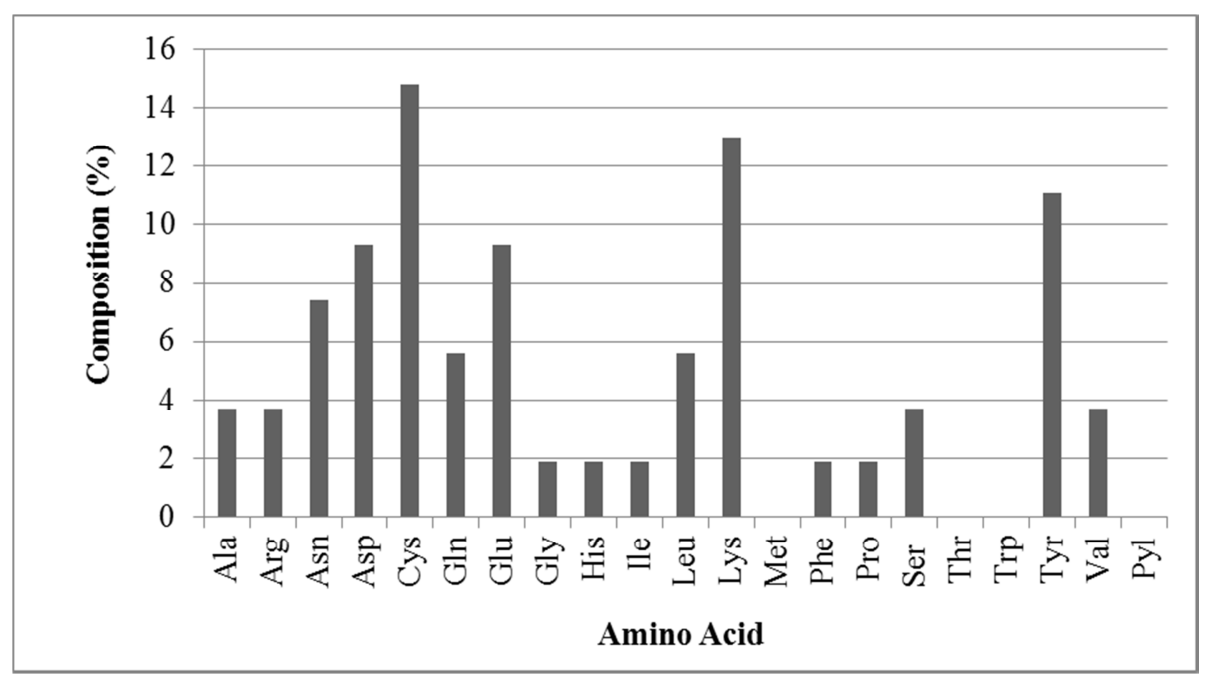

Figure 7. Amino acid composition of brazzein. 


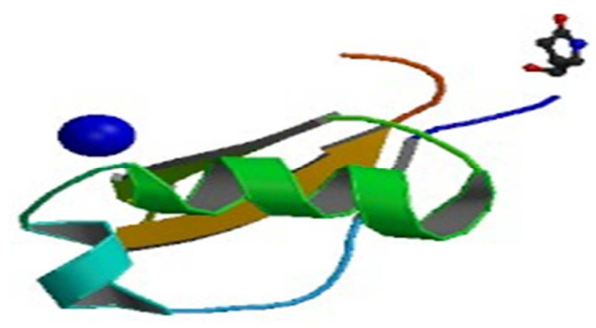

Figure 8. Structure of brazzein $4 H E 7$.

\section{Discussion}

The present work studied the hydrophobicity through carbon content on a number of brazzein mutants previously reported with focus on the two critical regions of the molecule suggested to be responsible for its sweetness $[1,8$, 9 , 15]. As revealed in the results, the two regions with residues 29-33 and 39-43 including residue 36 between these segments, exists a remarkable variation between brazzein mutants with the increased sweetness taste mainly at residues 29, 31 and 41 and mutants with reduced sweetness taste at residues 30, 33, 36 and 43. Mutation sites for brazzein mutants with increase in sweetness mainly at residues 29, 31 and 41 had the normal distribution curve of the carbon content with highly increased carbon frequency peak compared to the wild-type, consequently stabilized the protein and maintained it functionality. The other mutant such as E41Q maintained carbon content value at 0.314 , the known value for a stable protein and being in agreement with established findings that a stable and ordered proteins have a normal distribution of its carbon content symmetrical to about $0.314[14,16]$.

On the other hand the local carbon distribution curve of brazzein mutants with decreased sweetness revealed quite different distribution curve compared to those for increased sweetness. These mutants at residue position 30,33 and 36 were characterized by broadened distribution curve for carbon content with decreased carbon frequency peak thus destabilized protein function in terms of its sweetness. Broadened carbon distribution curve with decreased frequency peak destabilizes the structure and possibly leading to loss of protein functionality $[12,14]$. Based on carbon distribution analysis on several numbers of proteins, it has previously been reported that at mutation site, a single mutation can alter the protein function [17, 18]. Brazzein mutants at residue 43 (R43A and R43E) not only had a decreased carbon frequency peak but had also carbon content distribution curve shifting away from the normal value 0.314 which destabilized the local structure. Mutation can affect protein stability, protein function and protein-protein interactions as well as protein expression and sub cellular localization [19, 20]. Analysis through carbon content at mutation sites and its valuable in examining the stability and functions of mutant proteins has also previously been reported in some mutated proteins [18, 21, 22].
Carbon is the main element that contributes to the hydrophobic interactions in proteins [23]. Protein sequence prefers to have $31.45 \%$ of total carbon content for its structure stability and activity $[16,24]$. In this study carbon content analysis along of protein sequences for brazzein mutants with increased sweetness had normal carbon content profile distribution around $31.45 \%$. This normal distribution is an indication that these brazzein mutants had a stable structures maintaining functionality of the protein regardless of the mutation. Nevertheless, the carbon distribution profile for brazzein mutants with reduced brazzein sweetness showed low carbon distribution for their sequences consequently might have affected the protein functions. Carbon content and distribution profile along in a protein sequence is important for understanding molecular biology of individual sequence and contribute majorly towards protein folding and function [23, 24]. Moreover a carbon content profile distribution method can also be used for prediction of active sites or binding sites from sequence since the a carbon rich portions of sequence (more than $31.45 \%$ of carbon) is considered as active sites [24, 25].

Several studies aimed at identifying sweet taste determinants in brazzein have previously been reported. Some of these studies using different approaches have reported that charge was important for producing sweetness in brazzein and indicated that mutating positive charges to neutral or negative charge significantly decreases the sweetness in brazzein [1]. Other studies have also reported that mutations decreasing the overall negative charge and/or increasing the positive charge favors sweet-tasting protein strength [11, 26]. Apart from the influence of charge for sweetness taste in brazzein, in this study the carbon content distribution at mutation site has elucidated the role of atomic structure in examining mutation in brazzein. With an increasing demand for natural sweetener several studies have explored brazzein structure for the design and synthesis sweet-tasting protein $[27,28,29]$. The finding from this study has provided new insights into the structure-function relationship of brazzein which can be useful for design and synthesis of sweet tasting protein.

\section{Conclusion}

This study has further contributed insight in examining the effect of mutation in brazzein by delineating the role carbon content in relation to protein stability and function after mutation. Variations in carbon content distribution at mutational sites and on protein sequences was revealed between brazzein's mutants with increased in sweetness taste and those with decreased sweetness taste. The carbon content variation account for stability of protein upon mutation. While mutants with increased sweetness taste are marked with increased carbon content frequency peak, the mutants with decreased sweetness have decreased carbon frequency peak that destabilized the local structure and probably leading to loss of protein's function. The hydrophobicity and 
hydrophilicity for the entire sequences of mutants with increased sweetness taste also balance fairly well at the optimum position of stable proteins. The unveiled information provided herein can be useful in designing more effective brazzein analogs as feasible future sweeteners.

\section{References}

[1] Jin, Z., V. Danilova, F.M. Assadi-Porter, D.J. Aceti, J.L. Markley and G. Hellekant, 2003. Critical regions for the sweetness of brazzein. FEBS Lett., 544: 33-37.

[2] Nagata, K., N. Hongo, Y. Kameda, A. Yamamura, H. Sasaki, W. C. Lee and M. Tanokura, 2013. The structure of brazzein, a sweet-tasting protein from the wild African plant Pentadiplandra brazzeana. Acta Crystallogr. Sect. D-Biol. Crystallogr., 69(4): 642-647.

[3] Ming, D. and G. Hellekant, 1994. Brazzein, a new highpotency thermo stable sweet protein from Pentadiplandra brazzeana B. FEBS letters, 355(1):106-108.

[4] Hellekant, G. and V. Danilova, 2005. Brazzein a Small, Sweet Protein: Discovery and Physiological Overview. Chem. Senses, 30:88-89.

[5] Faus, I., 2000. Recent developments in the characterization and biotechnological production of sweet-tasting proteins. Appl. Microbiol. Biotechnol., 53: 145-251.

[6] Nookaraju, A., C.P. Upadhyaya, S.K. Pandey, K.E. Young, S.J. Hong, S.K. Park and S.W. Park, 2010. Molecular approaches for enhancing sweetness in fruits and vegetables. SciHortic., 127(1) 1-15.

[7] Yount, N.Y. and M.R. Yeaman, 2004. Multidimensional signatures in antimicrobial peptides. Proc. Natl. Acad. Sci. U.S.A. 101:7363-7368.

[8] Assadi-Porter, F.M., D.J. Aceti and J.L. Markley, 2000. Sweetness determinant sites of brazzein, a small, heat-stable, sweet-tasting protein. Arch Biochem Biophys. 376(2): 259-265.

[9] Assadi-Porter, F.M., E.L. Maillet, J.T. Radek, J. Quijada, J.L. Markley and M. Max, 2010. Key amino acid residues involved in multi-point binding interactions between brazzein, a sweet protein, and the T1R2-T1R3 human sweet receptor. J. Mol. Biol, 398(4): 584-599.

[10] Yoon, S.Y., J.N. Kong, D.H. Jo and K.H. Kong, 2011. Residue mutations in the sweetness loops for the sweet-tasting protein brazzein. Food Chem., 129(4): 1327-1330.

[11] Lee, J.W., J.E. Cha, D.H. Jo and K.H. Kong, 2013. Multiple mutations of the critical amino acid residues for the sweetness of the sweet-tasting protein, brazzein. Food Chem., 138(2): $1370-1373$.

[12] Rajasekaran, E., K. Akila and M. Vijayasarathy, 2011. Allotment of carbon is responsible for disorders in proteins. Bioinformaution, 6(8): 291.

[13] Rajasekaran, E., 2012. CARd: Carbon distribution analysis program for protein sequences. Bioinformation, 8(11): 508.

[14] Akila, K., N. Sneha and E. Rajasekaran, 2012. Study on carbon distribution at protein regions of disorder. Intern. J. Biosc., Biochem And Bioinf., 2(2): 58-60.
[15] Caldwell, J.E., F. Abildgaard, Ž. Džakula, D. Ming, G. Hellekant, and J.L. Markley, 1998. Solution structure of the thermostable sweet-tasting protein brazzein. Nat Struct. Mol. Biol, 5(6): 427-431.

[16] Rajasekaran, E. and M. Vijayasarathy, 2011. CARBANA: Carbon analysis program for protein sequences. Bioinformation, 5(10): 455.

[17] Zelko, I.N., T.J. Mariani and R. J. Folz, 2002. Superoxide dismutase multigene family: a comparison of the $\mathrm{Cu} \mathrm{Zn}-\mathrm{SOD}$ (SOD1), Mn-SOD (SOD2), and EC-SOD (SOD3) gene structures, evolution, and expression. Free Radic Biol Med., 33(3): 337-349.

[18] Amri, E., F.A. Mamboya, P.D. Nsimama and E. Rajasekaran, 2012. Role of carbon in crystal structures of wild-type and mutated form of dihydrofolate reductase-thymidylate synthase of plasmodium falciparum. Inter. J. Appl. Biol. Pharmaceut Tech. 3(3): 1-6.

[19] Yue, P., Z. Li, and J. Moult, 2005. Loss of protein structure stability as a major causative factor in monogenic disease. J. Mol. Biol., 353(2): 459-473.

[20] Reva, B., Y. Antipin and C. Sander, 2011. Predicting the functional impact of protein mutations: Application to cancer genomics. Nucleic Acids Res., 1: 39(17): e118.

[21] Mamboya, F.A., P.D. Nsimama, E. Amri, J.S. Sharmila and E. Rajasekaran, 2012. Carbon distribution analysis on mutations responsible for Li-Fraumeni syndrome.GSTF J. Bio Sci., 1(2): $1-6$.

[22] Nsimama, P.D., A.F. Mamboya, E. Amri and E. Rajasekaran, 2012. Correlation between the mutated colour tunings and carbon distributions in Luciferase bioluminescence. J. Comput. Intel. Bioinf., 5(2): 106-112.

[23] Johri, P., 2013. Atomic level sequence analysis-A Review. Int. J. Comput Bioinfo in Silico Model, 2(4): 173-179.

[24] Senthil, R. and E. Rajasekaran, 2009. Comparative analysis of carbon distribution and hydropathy plot. J. Adv. Biotech., 8(9): 30-31.

[25] Bragg, G.J. and L. Hyder, 2004. Nitrogen versus carbon use in prokaryotic genomes and proteomes. Biol. Sci., 271: 374-377.

[26] Esposito, V., R. Gallucci, D. Picone, G. Saviano, T. Tancredi and P.A. Temussi, 2006.The importance of electrostatic potential in the interaction of sweet proteins with the sweet taste receptor. J. Mol. Biol., 360(2): 448-456.

[27] Jo, H.J., J.S. Noh and K.H. Kong, 2013. Efficient secretory expression of the sweet-tasting protein brazzein in the yeast Kluyveromyces lactis. Protein expression and purification, 90(2): 84-89.

[28] Suh, J.Y., H.S. Kim, M.C. Kim and, K.H. Kong, 2014. Design and Evaluation of Synthetic Peptides Corresponding to the Sweetness Loop of the Sweet-Tasting Protein Brazzein. Bull. Korean Chem. Soc, 35(11): 3353.

[29] Fake, G. and J. Howard, 2014. Brazzein: A High-Intensity Natural Sweetener. In Commercial Plant-Produced Recombinant Protein Products (pp. 247-257). Springer Berlin Heidelberg. 